enhancement over some $\mathrm{TiO}_{2}$ silicate glasses. In this work, we continue studying these glasses using three different techniques to analyze the glass structures seeking to provide a deep insight for the relation between structure compositions and the optical characteristics of these glasses. The Radial Distribution Function analysis (RDF) combined with Raman spectroscopy is used to study these glasses. Positron annihilation life time spectroscopy and Doppler broadening measurements are carried out to investigate the change in the glass structure as the incorporation of $\mathrm{TiO}_{2}$ concentration into glass. The origin of the non-bridging oxygen (NBO) bonds has been traced correlating their existence with the measured nonlinear optical properties of the investigated glasses.

[1] M. Abdel-Baki, F. Abdel Wahab, F. El-Diasty, Mater. Chem. Phys. 96 [2006]201.

[2] Abou Shama A., Ph.D. Thesis, Ain Shams University (1995) 22-23.

\section{MS25 04}

Neutron diffraction, inelastic neutron scattering (INS) and DFT study of levoglucosan (1,6-anhydro- $\beta$-Dglucopyranose) Mariana Sládkovičová, Institute of Inorganic Chemistry, Slovak Academy of Sciences, Dúbravská cesta 9, SK-845 36 Bratislava, Slovak Republic. E-mail: Mariana.Sladkovicova@savba.sk

\section{Keywords: neutron diffraction, INS, DFT}

The study reported here is part of an ongoing project focused on obtaining an accurate description of hydrogen bonding in selected compounds using a combination of diffraction and spectroscopic methods with quantum chemical calculations. Neutron diffraction data are used to provide accurate information on the geometry of hydrogen bonds, while INS spectra are applied to obtain information on the dynamics of hydrogen atoms in the structure. Such information is then compared to theoretical calculation done for an isolated molecule with simulated surroundings and for crystal. The aim of the present study is to provide a full and accurate description of the hydrogen bonds in the structure of a monosaccharide levoglucosan (LG). The structure of LG was accurately determined by refinement of time-of-flight neutron single crystal diffraction data taken at 30 and $100 \mathrm{~K}[1,2]$. Molecules of LG are held together by a hydrogen bond array formed by a combination of strong O-H... $\mathrm{O}$ and supporting weaker C$\mathrm{H}$... O bonds. The strong hydrogen bonds link molecules in finite chains, with hydroxyl oxygens acting as both donors and acceptors of hydroxyl hydrogen atoms. The accurate specification of hydrogen bond geometry from neutron diffraction experiment allowed the structure to be correlated with DFT calculations and INS experiment. Hydrogen bonds influence the geometry of LG both, in vacuum [2] and in crystal structure [1] and their formation is reflected in the INS spectrum of crystalline LG [3]. The INS spectrum of LG is rather complex, characteristic for highly mixed modes. Most of the peaks are observed below $1500 \mathrm{~cm}^{-1}$. Here, characteristic $\mathrm{CH}$ and $\mathrm{OH}$ bending, torsional, and skeleton regions were defined, although there are no strict borders between them. In the higher parts of the spectrum only badly resolved $\mathrm{CH}$ and $\mathrm{OH}$ stretching modes appear (2800-3500 $\left.\mathrm{cm}^{-1}\right)$, mostly buried in intensive phonon wings. Wavelet analysis helped to localize the $\mathrm{OH}$ stretchings. Detailed interpretation of observed spectra and the assignment of peaks were accomplished by analyzing theoretically obtained spectra from periodic DFT calculations. Molecular dynamics calculations in solid state helped to interpret peaks in the lowest part of the experimental spectrum $\left(<200 \mathrm{~cm}^{-1}\right)$. Influence of hydrogen bonds on the spectrum was studied using molecular DFT approach. The calculations indicate strong frequency shifts due to $\mathrm{O}-\mathrm{H} . . . \mathrm{O}$ bonds and to noticeable extend due to weaker $\mathrm{C}-\mathrm{H}$...O contacts in bending and stretching regions. In the stretching region red shifts $\left(\sim 250 \mathrm{~cm}^{-1}\right)$ of $\mathrm{O}-\mathrm{H}$ and blue shifts $\left(\sim 30 \mathrm{~cm}^{-1}\right)$ of $\mathrm{C}-\mathrm{H}$ frequencies are predicted. The highest blue shifts $\left(\sim 300 \mathrm{~cm}^{-1}\right)$ are predicted for $\mathrm{OH}$ torsional modes.

[1] Smrčok L., Sládkovičová M., Langer V., Wilson Ch.C., Koóš M., Acta Cryst, 2006, B62, 912.

[2] Sládkovičová M., Smrčok L, Mach P.Rundlöf H., Cental Europ. J. Chem., 2007, 5, 1.

[3] Sládkovičová M., Smrčok L., Mach P., Tunega D., J. Mol. Structure, 2007, submitted. 